\title{
Docetaxel and oxaliplatin combination in second-line treatment of patients with advanced gastric cancer
}

\author{
Carlo Barone, Michele Basso, Giovanni Schinzari, Carmelo Pozzo, Nunziatina Trigila, \\ Ettore D'Argento, Michela Quirino, Antonio Astone, and Alessandra Cassano \\ Department of Medical Oncology, Università Cattolica del Sacro Cuore, Largo Agostino Gemelli n ${ }^{\circ}$ 8, 00168, Roma, Italy
}

\begin{abstract}
Background. In advanced gastric cancer few data are available on the efficacy or safety of new drug combination regimens after progression following first-line chemotherapy.

Methods. Patients with histologically confirmed advanced gastric cancer and Eastern Cooperative Oncology Group (ECOG) performance status (PS) less than 2, progressing after first-line chemotherapy, were eligible. Patients were treated with docetaxel $75 \mathrm{mg} / \mathrm{m}^{2}$ on day 1 and oxaliplatin $80 \mathrm{mg} / \mathrm{m}^{2}$ on day 2, every 3 weeks, until progression or unacceptable toxicity. Results. Between May 2002 and April 2005, 38 patients were enrolled. Men accounted for $73.7 \%$ of the patients and the median age was 59 years. The primary tumor was not resected in $47.4 \%$ of the patients; the peritoneum was the most frequent metastatic site $(60.5 \%)$. The first-line treatment was cisplatin, epirubicin, and infusional 5-fluorouracil (ECF) in $81.5 \%$ of the patients and cisplatin and infusional 5-fluorouracil (CF) in $15.7 \%$. The median number of cycles was 4.3 . The treatment was well tolerated, with no toxic deaths. National Cancer Institute (NCI) grade III-IV neutropenia was frequent $(26.3 \%)$, but no febrile neutropenia was reported. Severe asthenia $(15.7 \%)$ and severe nausea $(15.7 \%)$ required dose reductions in 2 patients and treatment discontinuation in another. The overall response rate was $10.5 \%$, and 18 patients $(47.3 \%)$ experienced disease stabilization ( 7 of them with significant clinical benefit). Median time to progression was 4.0 months (range, 2-8 months) and median overall survival was 8.1 months (range, 3-26 months). Thirteen patients $(34.2 \%)$ also received third-line chemotherapy, with an irinotecancontaining regimen, and their median overall survival was higher than that of the other patients (16.3 vs 6.0 months) Conclusion. The combination of oxaliplatin and docetaxel shows only marginal activity as second-line treatment, but it has a good tolerability profile. This suggests that there is room for optimizing the schedule as well as for planning sequential treatments in gastric cancer.
\end{abstract}

Key words Oxaliplatin · Docetaxel · Gastric cancer · Secondline therapy

Offprint requests to: C. Barone

Received: December 4, 2006 / Accepted: March 5, 2007

\section{Introduction}

Despite its decline in Western countries, gastric cancer remains the world's second leading cause of cancer mortality after lung cancer, accounting for approximately $12 \%$ of all cancer deaths [1,2]. Radical surgery remains the only possibility of cure, though prognosis appears to be poor even for resected patients [3]; indeed, the 5 -year median overall survival for resected patients is about $30 \%$. For this reason several trials, involving adjuvant and neoadjuvant treatments, are currently investigating the role of chemotherapy and radiotherapy [4]. Unfortunately, most patients relapse after surgery and many others are diagnosed when distant metastases are already present. For these patients only palliative chemotherapy can be offered.

In the 1990s three randomized trials of combination chemotherapy demonstrated benefits in terms of overall survival and quality of life in comparison with best supportive care [5-7]. The combination of 5-fluorouracil (5-FU), doxorubicin, and mitomycin-C (FAM) was considered the standard regimen until the FAMTX schedule (including 5-FU, doxorubicin, high-dose methotrexate, and leucovorin) was shown to be superior in terms of both response rates (41\% vs $9 \%)$ and overall survival (42 vs 29 weeks) [8]. On the other hand, cisplatin (CDDP)-based regimens demonstrated response rates of between $37 \%$ and $72 \%$ in phase II studies [9,10], although these results were not confirmed in phase III studies [11-13]. More recently, the ECF combination regimen of CDDP, epirubicin (EPI), and infusional 5FU proved very effective in phase II studies [14,15], and it was superior to FAMTX in terms of response rates (46\% vs $21 \% ; P=0.0002$ ) and overall survival (8.7 vs 6.1 months; $P=0.0005$ ) in a randomized phase III comparison, with mild toxicity (16). Since then the combination of CDDP and infusional 5-FU, with or without EPI, has become the most widely used regimen in front-line chemotherapy for advanced gastric cancer, even though 
some authors have reported no significant differences between CDDP and non-CDDP-containing regimens $[17,18]$. However, front-line chemotherapy for advanced gastric cancer has remained unsatisfactory, because overall survival does not generally exceed 10 months.

Great hope has been placed in new cytotoxic drugs, of which irinotecan (IRI), docetaxel (DTX), and 1oxaliplatin (OXA) seem the most promising. Both IRI and DTX have been shown to be active as first- or second-line therapy in combination with CDDP or 5FU in phase II studies [19-24]. A triple combination of DTX with CDDP and 5-FU proved significantly more effective than CDDP plus 5-FU, but overall survival was no more than 10.2 months, at the cost of higher toxicity [25]. On the other hand, the combination of IRI plus infusional 5-FU was superior to IRI plus CDDP in a randomized phase II study, and the combination was also shown to be no less effective than CDDP plus infusional 5-FU and to cause considerably less toxicity in a subsequent phase III trial $[20,21]$.

Only a few studies of OXA in the treatment of advanced gastric cancer are available. In the wake of the satisfactory results achieved in colorectal cancer, it was widely used in combination with 5-FU (FOLFOX regimen), and was also found to show some activity in CDDP-pretreated patients [26]. As front-line chemotherapy in phase II studies, this combination yielded response rates and overall survival of up to $40 \%$ and 10 months, respectively [27-29].

It has recently been shown that the combination of taxanes and OXA may be synergistic in vitro, when DTX is administered before OXA [30]; the same conclusion was reached in a phase I study [31]. Based on these data, we decided to evaluate a regimen that combined OXA and DTX in pretreated patients with advanced gastric cancer.

\section{Patients and methods}

This mono-institutional phase II study was conducted in accordance with the updated Declaration of Helsinki and with the rules of the local Ethics Committee.

\section{Patient selection}

Patients with histologically proven recurrent, locally advanced, or metastatic adenocarcinoma of the stomach or of the esophageal-gastric junction, not suitable for resection, were included. The patients also were required to be between 18 and 75 years of age, with ECOG performance status of 2 or less, to present bidimensionally measurable lesions according to WHO criteria, and to have a life expectancy of more than 3 months. Documented progression after at least one previous chemotherapy cycle was also required, while prior radiotherapy on the target lesion was cause for exclusion from the study. Patients with local recurrence were acceptable if they had at least one measurable affected lymph node; cytological or histological confirmation of metastasis was mandatory if only one metastatic lesion was present. All patients were required to have adequate hematological, renal, and hepatic function (hemoglobin $\geq 10 \mathrm{~g} / \mathrm{dl}$; neutrophils $\geq 2.0 \times 10^{9} / \mathrm{l}$; platelets [PLT] $\geq 150 \times$ $10^{9} / 1$; creatinine $\leq 1.25$ times the upper normal limit; aspartate aminotransferase [AST; SGOT] $\leq 2.5$ times the upper normal limits [UNL]; alanine aminotransferase [ALT; SGPT] $\leq 2.5$ times the upper normal limits; and total serum bilirubin $\leq 1.25$ times the upper normal limits; in those with liver metastasis: AST $\leq 5$ times the UNL, ALT $\leq 5$ times the UNL, and total serum bilirubin $\leq 1.5$ times the UNL). Written informed consent was required from each patient.

Exclusion criteria included: gastric or esophageal cancer other than adenocarcinoma; central nervous system metastases; unresolved bowel obstruction or sub-obstruction; chronic diarrhea; other serious medical conditions (unstable cardiac disease requiring treatment; uncontrolled angina pectoris; myocardial infarction within 6 months; active uncontrolled infections, uncontrolled diabetes with symptomatic peripheral neuropathy); any contraindication for DTX and OXA chemotherapy regimen; and history of other cancer, except for curatively treated nonmelanoma skin cancer or in-situ carcinoma of the cervix.

\section{Treatment and study parameters}

In the 2 days preceding the first course of chemotherapy, and before each cycle, patients underwent a complete physical examination and laboratory tests, including blood count, serum chemistry, liver enzymes, urinalysis, and serum carcinoembryonic antigen (CEA). Blood count determination was repeated weekly. Baseline tumor assessment with prospective identification of index lesion(s) was conducted not longer than 4 weeks before the enrollment and included abdomino-pelvic and lung computed tomography (CT) scans.

Chemotherapy consisted of: DTX $75 \mathrm{mg} / \mathrm{m}^{2}$ on day 1 as a $60-\mathrm{min}$ infusion and OXA $80 \mathrm{mg} / \mathrm{m}^{2}$ on day 2 as a 3-h infusion. The day before starting chemotherapy patients received intramuscular dexamethasone $8 \mathrm{mg}$ to prevent taxane-related allergic reactions; dexamethasone was continued every $12 \mathrm{~h}$ for 2 days after DTX. Routine antiemetic prophylaxis, also including a 5hydroxytryptamine-3-receptor antagonist, was mandatory on day 1 and day 2 . Treatment was repeated every 3 weeks. 
Tumor response was assessed every three cycles by CT scan, according to WHO criteria. Chemotherapy was continued until disease progression, unacceptable toxicity, or the patient's refusal. In the case of disease progression, patients were treated with other drug combinations at the discretion of the investigators. Patients were considered evaluable for response if they had received a minimum of two cycles of the treatment. Patients who progressed before two cycles were also considered evaluable.

Clinical benefit was assessed through a trial-specific pain checklist that was completed at baseline and every two cycles thereafter while patients were enrolled in the study; patients also recorded daily data on analgesic consumption and kept a weekly record of pain intensity on a 100-mm linear analog scale.

\section{Toxicity assessment and management}

Toxicity was assessed according to the National Cancer Institute (NCI) common toxicity criteria. Oxaliplatin (OXA)-related sensory neuropathy was scored as follows: grade 0 , no toxicity; grade I, mild paresthesia, loss of deep tendon reflexes; grade II, moderate paresthesia and objective sensory loss (without functional impairment); grade III, functional impairment.

Chemotherapy was delayed until recovery if neutrophils decreased to less than $1.5 \times 10^{9} / 1$ or platelets decreased to less than $100 \times 10^{9} / 1$ or in case of mucositis or diarrhea greater than NCI grade I. Doses of DTX and OXA were reduced by $25 \%$ in case of NCI grade IV neutropenia, febrile neutropenia, NCI grade IV thrombocytopenia, NCI grade III-IV diarrhea and mucositis, or NCI grade IV nausea. In the case of grade II neuropathy, OXA was reduced by $25 \%$, while in cases of grade III neuropathy it was discontinued. Secondary prophylaxis of neutropenia with colony-stimulating factors was allowed.

\section{Statistical considerations}

The primary endpoint was the overall response rate (complete plus partial response). Patients were recruited using a two-step phase II design according to Simon et al. [32]. If no objective response was observed in the first 14 patients, a response rate greater than $15 \%$ could be excluded with $95 \%$ accuracy and recruitment would be discontinued. In the event of at least one partial response, more than 30 patients would need to be recruited to determine an accurate response rate.

Secondary endpoints were time to progression and overall survival. Time to progression was calculated from the start of chemotherapy to the date of progression, and survival was defined as the interval time between recruitment and death. The Kaplan-Meier method was used to analyze time to progression and survival. All data have been updated to April 2006.

\section{Results}

\section{Patients}

Between May 2002 and April 2005, 38 consecutive eligible patients from a single institution were enrolled in the study. Their characteristics are summarized in Table 1 . The median age of the patients $(28$ men and 10 women) was 59 years (range, $33-75$ years). Nineteen $(50.0 \%), 12(31.5 \%)$, and $7(18.5 \%)$ had an Eastern Cooperative Oncology Group (ECOG) performance status of 0,1 , and 2, respectively. Many of them had not had surgery for primary tumor $(47.4 \%)$. For 10 of the

Table 1. Patient characteristics (38 patients)

\begin{tabular}{lcl}
\hline $\begin{array}{l}\text { Median age (years) } \\
\text { Sex }\end{array} \quad 59$ & (Range, 33-75) \\
$\quad$ Male & 28 & $(73.7 \%)$ \\
$\quad$ Female & 10 & $(26.3 \%)$ \\
Performance status (ECOG) & & \\
$\quad 0$ & 19 & $(50.0 \%)$ \\
1 & 12 & $(31.5 \%)$ \\
2 & 7 & $(18.5 \%)$ \\
Surgery for primary tumor & 20 & $(52.6 \%)$ \\
Sites of disease & 10 & $(26.3 \%)$ \\
$\quad$ Liver and nodes & 23 & $(60.5 \%)$ \\
$\quad$ Peritoneum & 8 & $(21.0 \%)$ \\
$\quad$ Lung metastases & 9 & $(23.6 \%)$ \\
$\quad$ More than 3 organs & 31 & $(81.5 \%)$ \\
Previous chemotherapy regimens & 6 & $(15.7 \%)$ \\
$\quad$ ECF & 1 & $(2.6 \%)$ \\
$\quad$ Cisplatin and 5-FU & 7.7 Months & \\
$\quad$ IRI and 5-FU & & \\
Median time to progression (front-line) & & \\
\hline
\end{tabular}


38 patients $(26.3 \%)$, the liver or lymph nodes were the only site of metastasis, while the peritoneum was the most frequent metastatic site in our series (23 patients; $60.5 \%$ ). Peritoneal carcinosis was the only site of disease in 5 patients. Eight patients $(21.0 \%)$ had lung metastases and in almost one-quarter of the patients $(23.6 \%)$ more than three organs were affected by disease.

All patients had failed to respond to at least one previous chemotherapy regimen. First-line treatment was a combination regimen of CDDP, EPI, and 5-FU (ECF) for most patients $(31 ; 81.5 \%)$; only 1 patient $(2.6 \%)$ had received IRI plus 5-FU and the other 6 $(15.7 \%)$ patients had been treated with a combination of CDDP and 5-FU (CF). Three of these 6 patients had disease progression 3-4 months after adjuvant chemotherapy with CDDP and 5-FU; for these patients, DTX and OXA was the first-line treatment for advanced disease. Overall median time to progression after starting the first-line treatment was 7.7 months (range, 2-17 months)

\section{Drug delivery and toxicity}

A total of 164 cycles of DTX and OXA were administered to the 38 patients, with a median number of cycles administered per patient of 4.3 (range, 1-8). Dose reduction was required in 7 cycles (out of 164), but no delay in the start of chemotherapy was required.

Dose intensity of both OXA and DTX was $98.9 \%$. The median cumulative dose of OXA was $344 \mathrm{mg} / \mathrm{m}^{2}$.

The treatment was well tolerated and no toxic death occurred. The incidence of the main toxicities is summarized in Table 2. The most common hematological

Table 2. Adverse effects according to NCI-CTC

\begin{tabular}{|c|c|c|c|c|}
\hline \multirow[b]{2}{*}{ Toxicity (38 patients) } & \multicolumn{2}{|c|}{ Grade $1-2$} & \multicolumn{2}{|c|}{ Grade 3-4 } \\
\hline & $n$ & $\%$ & $n$ & $\%$ \\
\hline \multicolumn{5}{|l|}{ Hematological } \\
\hline Neutropenia & 19 & 50.0 & 10 & 26.3 \\
\hline Thrombocytopenia & 2 & 5.2 & - & - \\
\hline Febrile neutropenia & - & - & - & - \\
\hline Anemia & 8 & 21.0 & - & - \\
\hline \multicolumn{5}{|l|}{ Gastrointestinal } \\
\hline Nausea/vomiting & 20 & 52.6 & 6 & 15.7 \\
\hline Diarrhea & 6 & 15.7 & - & - \\
\hline Constipation & 7 & 18.4 & - & - \\
\hline Stomatitis & 4 & 10.5 & - & - \\
\hline \multicolumn{5}{|l|}{ Oxaliplatin-related } \\
\hline Neuropathy/neurotoxicity & 16 & 42.1 & 1 & 2.6 \\
\hline Allergic reactions & - & - & - & - \\
\hline Thrombosis & 1 & 2.6 & - & - \\
\hline \multicolumn{5}{|l|}{ Other } \\
\hline Asthenia & 13 & 34.2 & 6 & 15.7 \\
\hline
\end{tabular}

side effect was neutropenia, though it was of grade 3-4 in only 10 patients $(26.3 \%)$, while there were no recordings of febrile neutropenia, severe thrombocytopenia, or severe anemia. Eight patients $(21.0 \%)$ and 2 patients (5.2\%) experienced grade 2 anemia and grade 1-2 thrombocytopenia, respectively.

The most important nonhematological toxicities were grade 3 nausea/vomiting and grade 3 asthenia, both occurring in 6 patients $(15.7 \%)$. When these two side effects were simultaneously present (2 patients) quality of life was significantly affected, and a $25 \%$ dose reduction was introduced. The same combination of toxic effects caused 1 patient to refuse a dose reduction and to withdraw from the study. Grade 1-2 nausea was fairly frequent (20 patients; $52.6 \%$ ), but was easily controlled with the appropriate antiemetic therapy. Grade 1-2 asthenia was also common (34.2\% of patients). Gastrointestinal side effects other than nausea and vomiting were rare: $6(15.7 \%)$ and 4 patients $(10.5 \%)$ experienced grade 1-2 diarrhea and grade 1 stomatitis, respectively. Seven patients (18.4\%) had grade 1-2 constipation after chemotherapy, probably because of the high dosage of 5-hydroxytryptamine-3-receptor antagonists as antiemetic medication. During chemotherapy 1 patient (2.6\%) developed a deep venous thrombosis of the left leg.

Oxaliplatin (OXA)-related peripheral sensory neuropathy was a common side effect but it was generally mild, probably due to the low cumulative dose of OXA. Fourteen patients $(36.8 \%)$ had grade 1 neuropathy and 2 patients $(5.3 \%)$, grade 2 neurotoxicity. Only 1 patient experienced grade 3 neuropathy, after the sixth cycle of chemotherapy. No allergic reactions to OXA or DTX were recorded.

\section{Efficacy}

All patients received at least one cycle of chemotherapy and 37 were assessable for response; 1 patient refused further treatment after the first cycle of therapy. Evaluation of response, with $95 \%$ confidence intervals (CI), is reported in Table 3. No complete response was obtained; 4 patients had a confirmed partial response, with an overall response rate of $10.5 \%$ (95\% CI, 0.8-20.8). Eighteen patients experienced stabilization of the disease (47.3\%; 95\% CI, 32.5-64.8); the disease progressed in 15 patients $(39.4 \%)$. Among patients with only peritoneal disease, stabilization was the best response in 3 of 5 patients. Seven of 18 the patients with stable disease experienced a clear clinical benefit, with complete resolution of obstructive gastrointestinal symptoms (3 patients) and pain (3 patients) or resolution of hydronephrosis (1 patient).

The median baseline serum CEA level in the 7 patients with stable disease and clinical benefit (50.2 \pm 
Table 3. Evaluation of response ( 37 patients)

\begin{tabular}{lrc}
\hline & \multicolumn{2}{c}{ Patients } \\
\cline { 2 - 3 } & $n$ & $\%$ \\
\hline Complete response & - & \\
Partial response & 4 & $10.5(95 \% \overline{C I}, 0.8-20.8)$ \\
Stable disease with significant clinical benefit & 7 & $18.4(95 \% \mathrm{CI}, 6.3-31.5)$ \\
Stable disease & 11 & $28.9(95 \% \mathrm{CI}, 15.0-44.5)$ \\
Progressive disease & 15 & 39.4 \\
\hline
\end{tabular}

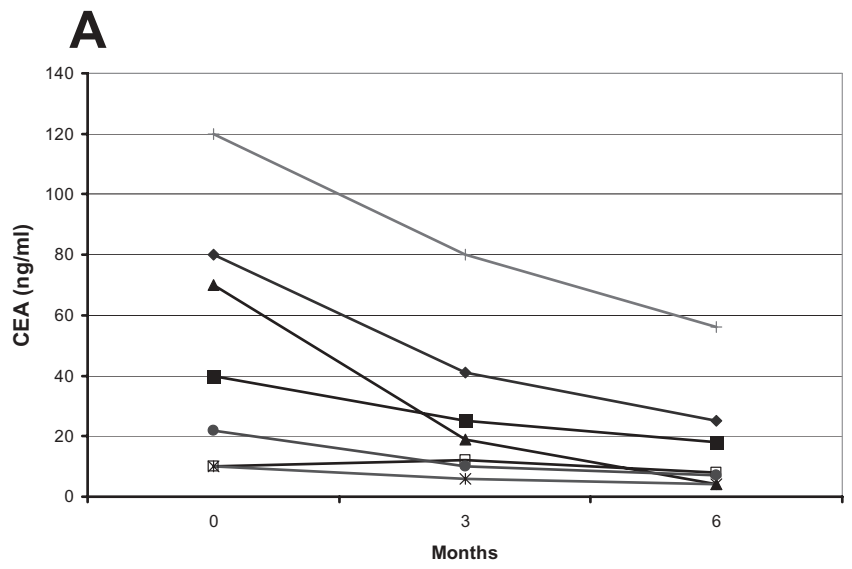

B

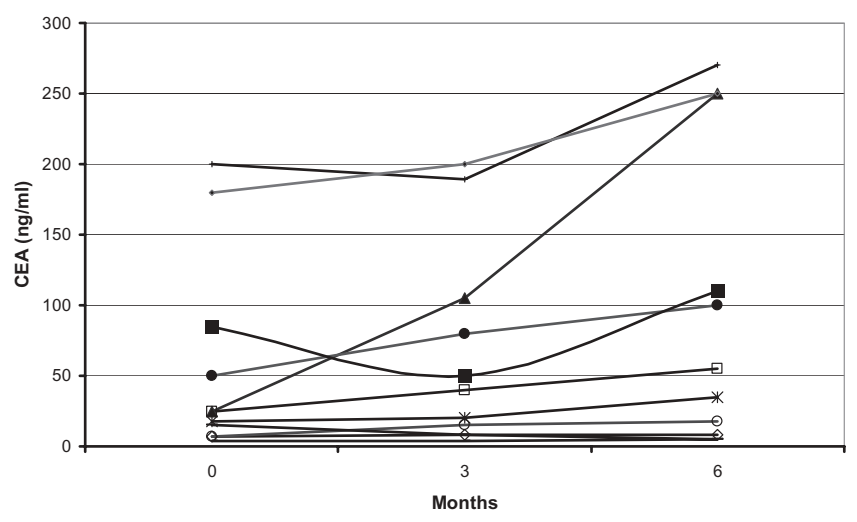

Fig. 1A,B. Trends of carcinoembryonic antigen $(C E A)$ during treatment. A Patients with stable disease and clinical benefit $(n=7)$; $\mathbf{B}$ remaining 11 patients with stable disease

$41.4 \mathrm{ng} / \mathrm{ml}$ ) remained unchanged or decreased after 3 and 6 months of treatment $(27.5 \pm 25.9 \mathrm{ng} / \mathrm{ml}$ and $17.4 \pm$ $21.9 \mathrm{ng} / \mathrm{ml}$, respectively; Fig. 1A). In comparison, the median baseline serum CEA level $(56.0 \pm 70.3 \mathrm{ng} / \mathrm{ml})$ in the other 11 patients with stable disease either remained unchanged or increased after $3(65.3 \pm 71.4 \mathrm{ng} / \mathrm{ml})$ and 6 months $(100.5 \pm 106.5 \mathrm{ng} / \mathrm{ml})$ of therapy, but in no patient was it decreased (Fig. 1B).

Median time to progression was 4.0 months (range, 2-8 months) and median overall survival was 8.1 months

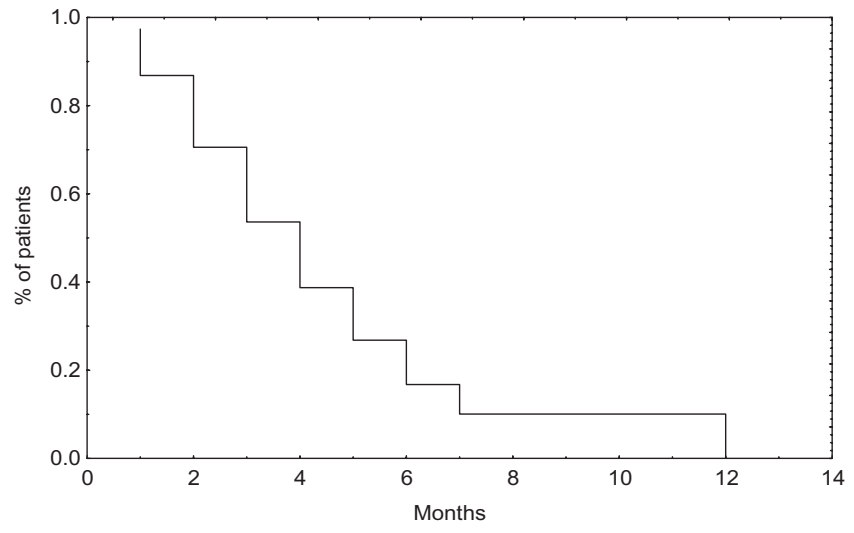

Fig. 2. Time to progression

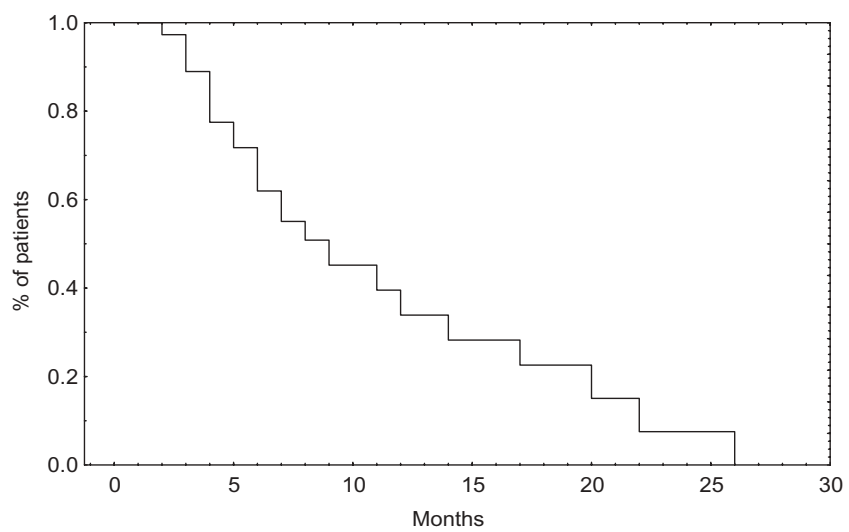

Fig. 3. Overall survival

(range, 3-26 months), as reported in Figs. 2 and 3, respectively.

Thirteen patients $(34.2 \%)$ maintained a good performance status PS at the end of second-line treatment; 1 of these had recorded a PR; 5, SD; and 7, PD during DTX and OXA. They were given third-line chemotherapy with a regimen containing IRI plus continuous infusion 5-FU, as previously reported [20]. The median overall survival of patients receiving third-line chemotherapy was 16.3 months, while the remaining 25 patients recorded a median overall survival of 6.0 


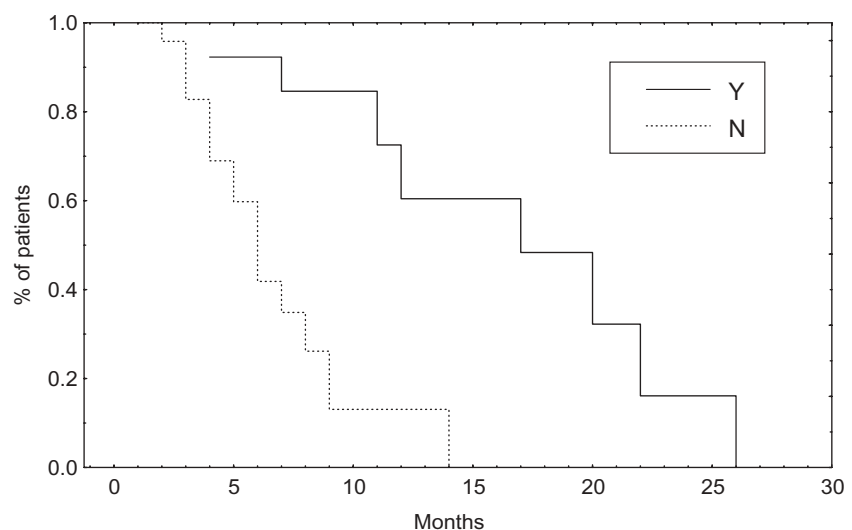

Fig. 4. Overall survivals of patients receiving third-line chemotherapy $(Y)$ and patients who did not receive out-of-study treatment $(N)$

months (Fig. 4); this difference was statistically significant $(P=0.00099)$.

\section{Discussion}

Despite the large number of clinical trials and combination treatments evaluated, the chemotherapy of advanced gastric cancer remains unsatisfying. In phase III studies the median survival does not exceed 10 months, even with combination therapies including new drugs such as docetaxel (DTX) [11-13,15,16,25]. These poor results are often obtained at the cost of a high incidence of hematological toxicity and diarrhea [25]. A possible strategy for improving the overall survival of patients with advanced gastric cancer could be an effective second-line therapy. A longer time to progression after second-line therapy might allow more patients to preserve a good performance status (PS) and receive further treatments. However, because the main therapeutic goal for most of these patients is palliation, second-line therapy has to be tolerable. We therefore studied a combination of DTX, which has established activity in advanced gastric cancer, and OXA, a platinum derivative whose adducts are not recognized by the mismatch repair (MMR) protein complex. In preliminary studies OXA yielded encouraging results in advanced gastric cancer in combination with infusional 5-FU, and was well tolerated [27-29]. Regimens and doses were selected on the basis of a previous phase I study in which this combination was investigated in patients affected by advanced breast or lung cancer [31].

The most important result of our study is the high tolerability of the regimen, even though most patients had previously been treated with combination therapy including cisplatin (CDDP), and 50\% of them had a PS of more than zero. No toxic death was observed, and the incidence of NCI-CTC grade 3-4 toxicity was very low. Only $26.3 \%$ of patients experienced NCI grade 3-4 neutropenia, with no febrile neutropenia or neutropenic infections. Hematological toxicity, other than neutropenia, was rare and never greater than grade 2. Among nonhematological toxicities, both grade 3 nausea/vomiting and grade 3 asthenia were experienced by $15.7 \%$ of patients and required a dose reduction in only two patients on account of their simultaneous occurrence. Oxaliplatin (OXA)-related sensory neuropathy was not an important concern; although mild neurotoxicity was common, only one patient experienced grade 3 cumulative neuropathy during the last course of chemotherapy, before progression was documented. The favorable toxicity profile appears particularly advantageous in patients with advanced gastric cancer, whose quality of life can be severely affected by the superimposition of gastrointestinal side effects on the diseaserelated symptoms.

The activity of our regimen is comparable to that described for second-line DTX monotherapy or combination regimens, as reported mostly in non-CDDP pretreated patients [33-35]. Although a response rate of $10.5 \%$ is both low and lower than those reported in other phase II second-line studies with DTX or OXAcontaining regimens, a time to progression of 4.0 months is comparable to that reported in the same studies. This discrepancy might be related to the significant and durable relief of symptoms in some patients with stable disease. Furthermore, more than $60 \%$ of patients in our study had peritoneal metastases, whose shrinkage according to WHO criteria is difficult to demonstrate. In effect, the simultaneous occurrence of relief of symptoms, a decrease in tumor markers, and stable disease cannot be unrelated to the action of chemotherapy, although it cannot be classified as a partial response. In agreement with these considerations, the median overall survival time of 8.1 months was longer than expected for a second-line chemotherapy in advanced gastric cancer and is even comparable to that obtained in some front-line studies $[11,12,15,23,26]$. In our opinion, the median overall survival time achieved with our treatment could depend on the high level of tolerability of our treatment regimen, as well as on the possibility of third-line chemotherapy in a subset of patients. In fact, although only $34.2 \%$ of patients received further treatment with an IRI-containing regimen, their median survival reached 16.3 months. This could be the consequence of a selection of long survivors, but also suggests that sequential treatments are possible in advanced gastric cancer, provided they are tolerable and not crossresistant. In effect, the combination of IRI with infusional 5-FU has recently been demonstrated to be as effective as CDDP plus infusional 5-FU, but better tolerated [21]. 
In conclusion, the combination of OXA and DTX is active to some extent as a second-line treatment of patients with advanced gastric cancer. The activity in a population of CDDP-pretreated patients is similar to that obtained with other DTX combinations in nonCDDP-pretreated patients. Interestingly, the combination of OXA and DTX is well tolerated and enables the administration of sequential treatments, which, in other gastrointestinal malignancies, have brought about a significant prolongation of overall survival rates [36-38]. This finding does not exclude the need not only to optimize the combination of OXA and DTX but also to search for more active first-line combinations and welltolerated sequences.

\section{References}

1. Parkin DM. Global cancer statistics in the year 2000. Lancet Oncol 2001;2:533-3.

2. Kelley JR, Duggan JM. Gastric cancer epidemiology and risk factors. J Clin Epidemiol 2003;56:1-9.

3. Macdonald JS. Treatment of localized gastric cancer. Semin Oncol 2004;314:566-73.

4. Hu JK, Chen ZX, Zhou ZG, Zhang B, Tian J, Chen JP, et al. Intravenous chemotherapy for resected gastric cancer: metaanalysis of randomized controlled trials. World J Gastroenterol 2002;8:1023-8.

5. Glimelius B, Ekstrom K, Hoffman K, Graf W, Sjoden PO, Haglund U, et al. Randomized comparison between chemotherapy plus best supportive care with best supportive care in advanced gastric cancer. Ann Oncol 1997;8:163-8.

6. Murad A, Santiago F, Petroianu A, Rocha PR, Rodriguez MA, Rausch M. Modified therapy with 5-fluorouracil, doxorubicin and methotrexate in advanced gastric cancer. Cancer 1993;72: 37-41.

7. Pyrrhonen S, Kuitenen T, Nyandoto P, Kouri M. Randomized comparison of fluorouracil, epidoxorubicin and methotrexate (FEMTX) plus supportive care with supportive care alone in patients with non-resectable gastric cancer. Br J Cancer 1995;71: 587-91.

8. Wils JA, Klein HO, Wagener DJ, Bleiberg H, Reis H, Korsten F, et al. Sequential high dose methotrexate and fluorouracil combined with doxorubicin - a step ahead in the treatment of advanced gastric cancer: a trial of the European Organization for Research and Treatment of Cancer Gastrointestinal Tract Cooperative Group. J Clin Oncol 1991;9:827-31.

9. Ychou M, Astre C, Rouanet P, Fabre JM, Saint-Aubert B, Domergue J, et al. A phase II study of fluorouracil, leucovorin and cisplatin (FLP) for metastatic gastric cancer. Eur J Cancer 1996;32A: 1933-7.

10. Preusser P, Wilke H, Achterrath W, Fink U, Lenaz L, Heinicke A, et al. Phase II study with the combination etoposide, doxorubicin and cisplatin in advanced measurable gastric cancer. J Clin Oncol 1989;7:1310-7.

11. Cocconi G, Bella M, Zironi S, Algeri R, Di Costanzo F, De Lisi $\mathrm{V}$, et al. Fluorouracil, doxorubicin and mitomycin combination versus PELF chemotherapy in advanced gastric cancer: a prospective randomized trial of the Italian Oncology Group for Clinical Research. J Clin Oncol 1994;12:2687-93.

12. Kelsen D, Atiq OT, Saltz L, Niedzwiecki D, Ginn D, Chapman D, et al. FAMTX versus etoposide, doxorubicin and cisplatin: a random assignment trial in gastric cancer. J Clin Oncol 1992;10: $541-8$.
13. Kim NK, Park YS, Heo DS, Suh C, Kim SY, Park KC, et al. A phase III randomized study of 5-fluorouracil and cisplatin versus 5-fluorouracil, doxorubicin and mitomycin $\mathrm{C}$ versus 5-fluorouracil alone in the treatment of advanced gastric cancer. Cancer 1993;71: 3813-8.

14. Cascinu S, Labianca R, Alessandroni P, Marcellini M, Silva RR, Pancera $\mathrm{G}$, et al. Intensive weekly chemotherapy for advanced gastric cancer using fluorouracil, cisplatin, epi-doxorubicin, 6Sleucovorin, glutathione and filgastrim: a report from the Italian Group for the Study of Digestive Tract Cancer. J Clin Oncol 1997;15:3313-39.

15. Webb A, Cunningham D, Scarffe JH, Harper P, Norman A, Joffe $\mathrm{JK}$, et al. Randomized trial comparing epirubicin, cisplatin and fluorouracil versus fluorouracil, doxorubicin and methotrexate in advanced esophagogastric cancer. J Clin Oncol 1999;80: 269-72.

16. Waters JS, Norman A, Cunningham D, Scarffe JH, Webb A, Harper $\mathrm{P}$, et al. Long term survival after epirubicin, cisplatin and fluorouracil for gastric cancer: results of a randomized trial. Br J Cancer 1999;80:269-72.

17. Vanhoefer U, Wagner T, Lutz M, van Cutsem E, Nordlinger B, Rause S, et al. Randomized phase II study of weekly $24 \mathrm{~h}$ infusion of high dose 5-FU+/- folinic acid (HD-FU+/-FA) versus HDFU/FA/biweekly cisplatin in advanced gastric cancer. EORTC trial 40953. Eur J Cancer 2001;37(Suppl 6):S27(abstract 88).

18. Barone C, Corsi DC, Pozzo C, Cassano A, Fontana T, Noviello $\mathrm{MR}$, et al. Treatment of patients with advanced gastric carcinoma with a 5-fluorouracil-based or cisplatin-based regimen: two parallel randomized phase II studies. Cancer 1998;82:1460-7.

19. Fatatsuki K, Wakui A, Nakao I, Sakata Y, Kambe M, Shimada Y, et al. Late phase II study of irinotecan hydrochloride (CPT-11) in advanced gastric cancer. CPT-11 Gastrointestinal Cancer Study Group. Gan To Kagaku Ryoho 1994;21:1033-8.

20. Pozzo C, Barone C, Szanto J, Padi E, Peschel C, Bukki J, et al. Irinotecan in combination with 5-fluorouracil and folinic acid or with cisplatin in patients with advanced gastric or esophagealgastric junction adenocarcinoma: results of a randomized phase II study. Ann Oncol 2004;15:1773-81.

21. Dank M, Zaluski J, Barone C, Valvere V, Peschel C, Wenczl M, et al. Randomized phase 3 trial of irinotecan (CPT-11+5FU/folinic acid (FA) vs CDDP + 5FU in first-line advanced gastric cancer patients. J Clin Oncol 2005;23(S16):(abstract no. 4403).

22. Roth AD, Maibach R, Martinelli G, Fazio N, Aapro MS, Pagani $\mathrm{O}$, et al. Docetaxel (Taxotere)-cisplatin (TC): an effective drug combination in gastric carcinoma. Swiss Group for Clinical Cancer Research (SAKK) and European Institute of Oncology (EIO). Ann Oncol 2000;11:301-6.

23. Constenla M, Garcia-Arroyo R, Lorenzo I, Carrete N, Campos B, Palacios P. Docetaxel, 5-fluorouracil and leucovorin as treatment for advanced gastric cancer: results of a phase II study. Gastric Cancer 2002;5:142-7.

24. Ajani JA, Fodor M, Van Cutsem E, Tjulandin S, Moiseyenko V, Cabial F, et al. Multinational randomized phase II study of docetaxel (T) and cisplatin (C) with or without 5-flurouracil (FU) in patients (pts) with advanced gastric or GE junction adenocarcinoma (AGC-AGEJC). Proc Am Soc Clin Oncol 2000;19:247a (abstract 957).

25. Moiseyenko VM, Ajani JA, Tjulandin SA, Majlis A, Constenla $\mathrm{M}$, Boni C, et al. Final results of a randomized controlled phase III trial (TAX 325) comparing docetaxel (T) combined with cisplatin (C) and 5-fluorouracil (F) to CF in patients (pts) with metastatic gastric adenocarcinoma (MGC). J Clin Oncol 2005;23(S16): (abstract no. 4002).

26. Kim DY, Kim JH, Lee SH, Kim TY, Heo DS, Bang YJ, et al. Phase II study of oxaliplatin, 5-fluorouracil and leucovorin in previously platinum-treated patients with advanced gastric cancer. Ann Oncol 2003;14:383-7.

27. Al-Batran SE, Atmaca A, Hegewisch-Becker S, Jaeger D, Hahnfeld S, Rummel HJ, et al. Phase II trial of biweekly infusional 
fluorouracil, folinic acid and oxaliplatin in patients with advanced gastric cancer. J Clin Oncol 2004;22:658-63.

28. De Vita F, Orditura M, Matano E, Bianco R, Carlomagno C, Infusino $\mathrm{S}$, et al. A phase II trial of biweekly oxaliplatin plus infusional 5-fluorouracil and folinic acid (FOLFOX-4) as first line treatment of advanced gastric cancer patients. $\mathrm{Br} \mathrm{J}$ Cancer 2005;9:1644-9.

29. Louvet C, Andre T, Tigaud JM, Gamelin F, Douillard JY, Brunet $\mathrm{R}$, et al. Phase II study of oxaliplatin, fluorouracil and folinic acid in locally advanced or metastatic gastric cancer patients. J Clin Oncol 2002;20:4543-8.

30. Tanaka R, Ariyama H, Qin B, Takii Y, Baba E, Mitsugi K, et al. In vitro schedule-dependent interaction between paclitaxel and oxaliplatin in human cancer cell lines. Cancer Chemother Pharmacol 2005;55:595-601. Epub 2005 Feb 25.

31. Agelaki S, Kouroussis C, Mavroudis D, Kalbakis K, Souglakos J, Reppa D, et al. A phase I study of docetaxel (D) and oxaliplatin (L-OHP) as front-line treatment in metastatic breast cancer and non-small cell lung cancer (NSCLC). Proc Am Soc Clin Oncol 2000;19:abstract 443.

32. Simon R, Thall PF, Ellenberg SS. New designs for selection of treatments to be tested in randomized clinical trials. Stat Med 1994:13:417-29.

33. Kunisaki C, Imada T, Yamada R, Hatori S, Ono H, Otsuka Y, et al. Phase II study of docetaxel plus cisplatin as second-line combined therapy in patients with advanced gastric carcinoma. Anticancer Res 2005;25:2973-7.

34. Park SH, Kang WK, Lee HR, Park J, Lee KE, Lee SH, et al. Docetaxel plus cisplatin as second-line therapy in metastatic or recurrent advanced gastric cancer progressing on 5-fluorouracilbased regimen. Am J Clin Oncol 2004;27:477-80.

35. Polyzos A, Tsavaris N, Kosmas C, Polyzos K, Giannopoulos A, Felekouras E, et al. Subsets of patients with advanced gastric cancer responding to second-line chemotherapy with docetaxelcisplatin. Anticancer Res 2006;26:3749-53.

36. Tourningand C, Andrè T, Achille E, Lledo G, Flesh M, MeryMignard D, et al. FOLFIRI followed by FOLFOX 6 or the reverse sequence in advanced colorectal cancer: a randomized GERCOR study. J Clin Oncol 2004;15:229-37.

37. Rothenberg LM, Amit LA, Bigelow RH, Berlin JD, Marshall JL, Ramanathan RK, et al. Superiority of oxaliplatin and flurouracilleucovorin compared with either therapy alone in patients with progressive colorectal cancer after irinotecan and fluorouracilleucovorin: interim results of a phase III trial. J Clin Oncol 2003; 21:2059-69.

38. Grothey A, Sargent D, Goldberg RM, Schmoll HJ. Survival of patients with advanced colorectal cancer improves with the availability of fluorouracil-leucovorin, irinotecan and oxaliplatin in the course of treatment. J Clin Oncol 2004;22:1209-14. 